\title{
Structural Dynamics and Functional Analysis of Saprolegnia Parasitica Chitin Synthases 5 in a Phospholipid Bilayer
}

\author{
Sourav Biswas ${ }^{1}$, Md. Rubel Hossen ${ }^{1}$, Shaila Akter ${ }^{1}$, Md. Ackas Ali ${ }^{1}$, Mohammad A. \\ Halim $^{2}$, and Obayed Ullah $\mathrm{M}^{1}$ \\ ${ }^{1}$ Division of Infectious Diseases and Division of Computer-Aided Drug Design The \\ Red-Green Research Centre BICCB Tejgaon Dhaka Bangladesh \\ ${ }^{2}$ Kennesaw State University
}

January 29, 2022

\begin{abstract}
Saprolegnia parasitica is an oomycete responsible for fish disease called saprolegniosis, which poses economic and environmental burden on aquaculture production. Chitin is an essential cell wall component of S. parasitica synthesized by chitin synthase (CHS) enzyme. CHSs are large membrane-integrated enzymes with multiple domains responsible for cellular integration and activation. In saprolegnia, CHS5 of S. parasitica (SpCHS5) contains an N-terminal domain, a catalytic domain of the glycosyltransferase -2 family containing a GT-A fold, and a C-terminal transmembrane domain. In addition, SpCHS5 consists of the MIT (microtubule interacting and trafficking) domain which is crucial for intracellular trafficking of SpCHS5. There is no three-dimensional structure of SpCHS5 is reported yet disclosing the structural details of this protein. We have developed a structural model of full-length SpCHS5 and validated it by molecular dynamics. This model structure will assist on designing antiparasitic drug. Structural GT-A domain and transmembrane contribute stable alpha-helix where MIT domain demonstrate flexible conformation based on phosphorylation sites. From the chitin dynamic movement in the protein cavity, we predicted Asp442, Asp386, Ser541, Tyr379, Asp328, Gln481, Trp485, Tyr645, Thr641, Tyr177 residues as a prominent cavity lining site. Overall, this present work will be helpful to understand the biochemistry of CHS5 and design inhibitors against SpCHS5.
\end{abstract}

\section{Hosted file}

SpCHS5_manuscript_Willy.doc available at https://authorea.com/users/457994/articles/554608structural-dynamics-and-functional-analysis-of-saprolegnia-parasitica-chitin-synthases5-in-a-phospholipid-bilayer 